\title{
Tangence
}

\section{Les voyages de la plume (Petit commentaire devant servir à la construction pratique de la machine à explorer quelques romans de Paul Auster)}

\section{Richard Saint-Gelais}

Numéro 43, mars 1994

Babel et après : Paul Auster

URI : https://id.erudit.org/iderudit/025802ar

DOI : https://doi.org/10.7202/025802ar

Aller au sommaire du numéro

Éditeur(s)

Tangence

ISSN

0226-9554 (imprimé)

1710-0305 (numérique)

Découvrir la revue

Citer cet article

Saint-Gelais, R. (1994). Les voyages de la plume (Petit commentaire devant servir à la construction pratique de la machine à explorer quelques romans de Paul Auster). Tangence, (43), 68-79. https://doi.org/10.7202/025802ar 


\title{
Les voyages de la plume (Petit commentaire devant servir à la construction pratique de la machine à explorer quelques romans de Paul Auster)
}

\author{
Richard St-Gelais
}

Avec son nom multiplié dans les annuaires téléphoniques de chaque grande ville, Steinberg peut arriver sans difficulté à l'anonymat. Dans un édifice à appartements de New York où il a vécu, il y avait deux Steinberg sur le même étage, et à East Hampton, où il possède une maison, vit un autre Saul Steinberg. [...] Il y a quelques années, Steinberg a perdu patience et a téléphoné à son homonyme d'East Hampton.

"Est-ce que je parle à Saul Steinberg?", demanda-t-il.

“Oui ", lui répondit l'autre.

"Mais êtes-vous le vrai Saul Steinberg?" "Non", répondit le pauvre homme.

"En êtes-vous sûr?"

Harold Rosenberg ${ }^{1}$

On le dit, et c'est probablement juste: si l'écriture de Paul Auster fascine, cela tient en bonne part à ce qu'elle a de raréfié et de dense à la fois, comme si les phrases trompeusement simples qu'elle aligne ne faisaient jamais que délimiter une surface mais une surface qui se creuserait de tiroirs secrets, d'issues dérobées, de passages insoupçonnés. C'est que chaque texte d'Auster en cache un, ou plutôt toujours plusieurs autres, certains

1 Harold Rosenberg, Saul Steinberg, New York, Alfred Knopf/Whitney Museum of American Art, 1978, p. 13; ma traduction. 
signés Auster (ou, nous le savons maintenant, Benjamin), d'autres non, mais qui tous, au-delà de leur apparent disparate, entrent dans l'orbite de la constellation P. A. ${ }^{2}$ L'intertextualité, ici, est aux antipodes d'un name dropping littéraire: non pas un morceau de culture exhibé pour lui-même, mais quelque chose de plus dépouillé, de plus inquiétant aussi: chaque fois, une pièce qui s'ajoute au labyrinthe, sans que le lecteur ne sache trop, avant d'aller y voir, jusqu'à quel point elle ne démultiplierait pas les réseaux du texte qu'il a sous les yeux. Le paradoxe est ici que le texte parvient ainsi à annexer ce qu'intertextuellement il convoque, mais d'une façon telle que l'œuvre renonce irrémédiablement à revendiquer quelque identité que ce soit. En ce sens elle est, mais nous le savons déjà, exemplairement américaine.

Ainsi, des autours de Paul Auster, de nombreux lecteurs de City of Glass auront retenu, sans doute, une intrigante panoplie d'auteurs: Edgar Poe (pour "William Wilson"), Marco Polo (mark? Paul ${ }^{3}$ ), Montaigne, Defoe et Swift (pour leurs réflexions sur les enfants gardés en isolation), Cervantes (pour les multiples auteurs que se donne Don Quichotte), plusieurs autres encore, certains même fictifs - à commencer par Stillman, l'universitaire devenu un fou meurtrier.

À cet égard, le quatrième roman d'Auster, In the Country of Last Things ${ }^{4}$, semble à première vue faire un peu bande à part,

2 L'initiale, ou plutôt les initiales travaillent de toutes parts l'espace intertextuel austérien, y ménageant des parcours privilégiés, non pas en ce qu'elles livreraient une quelconque clé interprétative mais en ce qu'elles tracent les deux lignes de force d'un réseau indéfiniment diffracté : d'une part, la tentation constante pour un semi-anonymat et le mystère qu'il suscite; d'autre part, les rebondissements vers d'autres patronymes, d'autres identités. Ainsi, pour s'en tenir au " $P_{»}$ : $P$. A. bien sûr (Paul Auster, puis Peter Aaron dans Leviatban), P. B. (Paul Benjamin, puis, toujours dans Leviatban, Benjamin Sachs), P. S. (Peter Stillman dans City of Glass, mais aussi, partout, les post scriptutm de ces textes qui ne s'écrivent jamais qu'après, qu'à travers d'autres). Et puis, toujours sous-jacent mais jamais bien loin, le fameux P. I. de Private Investigator. Et on verra plus loin que ce n'est là que la pointe de l'iceberg...

3 Que P. A. soit passé maître dans l'art de susciter de ces "plagiats par anticipations" chers aux oulipiens, on en voit l'indice dans le fait que Marco Polo puisse, ne serait-ce que le temps d'une hypothèse déraisonnable, sembler un (autre) pseudonyme d'Auster...

4 Paul Auster, In the Country of Last Things, Londres, Faber \& Faber, 1989 [1987]. Ce roman a été traduit en français (par Patrick Ferragut) sous le titre Le voyage d'Anna Blume, Paris, Actes Sud, 1989. Dorénavant, sauf indication 
en ce que les références intertextuelles y sont, pour une fois, quasi absentes. La ville où débarque Anna Blume n'est pas précisément un lieu où les habitants ont beaucoup de temps à consacrer à la lecture; la bibliothèque, certes, contient une collection impressionnante de livres, mais que les résidents-chercheurs abandonnent au désordre, vendent pour de l'argent et même brûlent pour se tenir au chaud pendant l'hiver. Mais cette impression ne résiste pas à un examen plus attentif : le monde de In the Country est construit de mots hantés par d'autres mots, de noms hantés par d'autres noms; aussi Anna, lorsqu'elle avoue avoir mis au feu certains livres, ajoute aussitôt que c'était la seule façon pour elle et Sam de survivre, et donc de faire exister le livre sur lequel il travaillait depuis des mois (p. 116, 127). Voilà qui montre, à peine allusivement, à quel point l'intertextualité, loin de se réduire à une pratique confortable, compose avec la précarité des textes - à quel point toute reprise est aussi, incontournablement, un effacement.

Cette ambivalence foncière de l'intertextualité a, on s'en doute, des répercussions non négligeables sur la lecture. On sait par exemple à quel point les textes d'Auster s'ingénient à faire de leurs lecteurs des "fins limiers" 5 . Mais une importante différence mérite d'être signalée: tandis que les lecteurs fins limiers présupposés par les sciences cognitives se consacrent à la résolution de problèmes (surtout inférentiels ${ }^{6}$ ), les lecteurs d'Auster, eux, sont amenés à parcourir, vite en tous sens, de véritables labyrinthes de signes de telle sorte que le jeu, ou le travail, trouve moins sa justification dans une solution à débusquer que dans le plaisir même de l'exploration. Cela tient notamment à ce que la foison de coïncidences, recoupements et similitudes troublantes ne donne lieu, ni à un récit global qui subsumerait toutes les cellules fictionnelles, ni à un imaginaire homogène qui se profilerait derrière les textes.

L'instrument privilégié, ici, est assurément le nom propre (encore que le singulier, chez Auster, lui sied fort peu), assez

contraire, les chiffres entre parenthèses renverront respectivement à ces deux éditions.

5 Je reprends ici la judicieuse formule proposée par Daniel Vaillancourt dans "Litterrature et théories de la cohérence: un rendez-vous reporté", $R S / S I$, vol. $\mathrm{X}, \mathrm{n}^{\mathrm{os}} 1-2-3,1990$, p. $65-66$.

6 Comme le dit Vaillancourt: «Le fin limier cherche anxieusement à retrouver une représentation extensionnelle des faits discursifs qui lui servira à réguler la cohérence du discours ", op. cit., p. 65. 
insignifiant pour ne pas devenir le véhicule d'une thématique, assez référentiel, en même temps, pour que les connexions qu'il permet impliquent la possibilité d'une identification - mais aussi celle de son refus qui vient savamment brouiller les pistes. Les lecteurs sont ainsi amenés à se poser des questions à la fois ludiques et troublantes: y aurait-il un lien entre William, le frère d'Anna disparu sans laisser de traces, et William Wilson, pseudonyme de Quinn dans City of Glass ${ }^{7}$ ? Le "passeport d'un dénommé Quinn" qu'Anna a découvert lors de sa chasse aux objets (p. 36, 45) n'aurait-il pas été perdu par ce même Quinn ${ }^{8}$ ?

Les pistes de lecture deviennent proprement foisonnantes pour peu qu'on passe aux ressemblances ou aux parallélismes. Ainsi, et toujours en ne prenant que In the Country comme point de départ, la chute d'Anna et sa survie miraculeuse (p. 125, 137) trouveront un écho, dans Leviathan, dans celles de Benjamin Sachs, dont le nom arbore les mêmes initiales que Boris Stepanovich ${ }^{9}$, tout comme Anna Blume partage celles d'Alexander Berkman (toujours dans Leviathan; $c f$. p. 251), d'autant plus que ce dernier est l'auteur d'un... ABC of Communist Anarchism (id. ${ }^{10}$. De plus, Leviathan nous apprend que Berkman a assassiné Henry Clay Frick, richissime Américain qui a laissé son nom non seulement à un musée new-yorkais, mais aussi, fictivement cette fois et à rebours, à Otto Frick, l'homme à tout faire de Woburn House, dans In the Country ${ }^{11}$. Si on ajoute que le prénom de Victoria Woburn s'apparente à celui de Virginia Stillman (City of Glass) et que les initiales de Samuel Farr, en plus d'une allusion à la science-fiction,

7 Cf. Paul Auster, City of Glass in The New York Trilogy, New York, Penguin coll. "Contemporary American Fiction ", 1990 [1985], p. 3.

8 En fait, il est tentant de renverser ici les relations établies dans City of Glass en supposant que dans In the Country "Quinn" pourrait bien avoir été un nom d'emprunt de William. Voilà qui confirmerait que, pour l'écriture austérienne, il n'y a que des pseudonymes.

9 Ainsi que celles d'une certaine Beulah Stausky, personnage épisodique de In the Country (p. 176-190).

10 On pourrait en conclure qu'Anna Blume, A. B., symbolise quelque chose comme le commencement ou l'origine, si Otto Frick, qui a comme elle un prénom palindromique, ne disait à propos de leurs noms: "Sans fin nulle part mais ça recommence" (p. 133, 145).

11 J'ajoute que dans mon édition du Frommer's New York (New York, Simon \& Shuster, 1989, p. 170), ce musée est décrit comme une "oasis", terme qui s'applique manifestement aussi à la résidence Woburn elle-même. On ne s'étonnera donc pas que la mort de Frick (p. 172, 186) coïncide avec le début de la fin pour ce qui est des activités de la résidence. 
sont aussi celles de Sophie Fanshawe (The Locked Room), qui a son tour possède le même prénom que Sophie Calle, la dédicataire de Leviatban, on aura une idée de l'effervescence des renvois: sous la plume d'Auster, l'ici n'est jamais qu'un lieu textuel instable, sans cesse susceptible d'être déporté.

\section{La toponymie imaginaire}

Il ne faudrait pas oublier toutefois que In the Country, à la différence de plusieurs romans d'Auster qui reprennent (et problématisent) le modèle du récit de détection, s'insère dans un intertexte quelque peu différent: celui du roman d'anticipation. La relation entre texte et intertexte générique a cependant des chances de fonctionner autrement dans ce cas-ci, dans la mesure où le modèle science-fictionnel, pour maints lecteurs, n'est pas connu de première main, mais plutôt à travers les stéréotypes qui, inlassablement, dissuadent plusieurs d'aller voir ce qu'il en est.

Un indice parmi d'autres de la prégnance de ces stéréotypes se trouve dans le texte de présentation de l'édition britannique, dont le rédacteur anonyme s'est senti obligé de préciser que le "monde futuriste" dépeint dans In the Country projette son ombre sur le nôtre ("chillingly shadows our own"), comme si seul ce réalisme oblique pouvait sauver le roman du discrédit général qui frappe la science-fiction (à laquelle, de toute façon, on se garde bien de l'identifier formellement). Or c'est là réduire la portée du roman, et doublement: non seulement le roman peut-il se lire sans la lorgnette allégorisante qui consiste à rabattre le monde fictif sur le nôtre, mais de plus rien n'indique même que l'on ait affaire à une anticipation, à une extrapolation basée sur ce qui resterait notre monde. Ce qui frappe, au contraire, à lire avec moindrement d'attention, c'est le soin avec lequel le texte évite toute indication géographique ou chronologique qui puisse relier le monde fictif au nôtre ${ }^{12}$. Comment ne pas faire l'hypothèse, dès lors, qu'on a affaire, au sens fort, à un autre monde - et donc à

12 Les seules exceptions ne sont rencontrées que passablement loin dans le roman: l'Angleterre (p. 136, 148), Paris, la Russie et la Turquie (p. 146, 159), Minsk (p. 149, 163), la France (p. 152, 164). De plus, leur valeur indicative est essentiellement négative puisque chacun de ces lieux apparaît comme un ailleurs par rapport à la ville qui reste donc insituable. Quant à la chronologie, elle ne se raccroche à aucun événement de notre histoire. 
un monde dont il est impossible de déterminer a priori jusqu'à quel point il diverge de notre monde de référence?

Ne nous le cachons pas: les conséquences d'une telle décision peuvent surprendre. Ainsi, si les personnages qui apparaissent dans les récits de Boris Stepanovich (le duc de Fantômas et la comtesse Oblomov ${ }^{13}$ ) nous apparaissent un peu trop tapageusement empruntés à des fictions, ne nous hâtons pas d'en conclure qu'il s'agit là, simplement, d'une autre preuve que Boris est en train de fabuler: qu'est-ce qui dit que Fantômas et Oblomov ne sont pas, dans le monde fictif, des personnages bistoriques? Exclure a priori une telle hypothèse reviendrait à réduire singulièrement le champ des possibilités, non seulement de la science-fiction, mais de la fiction en général.

À qui pourrait répondre que de telles hypothèses relèvent davantage d'une fantaisie de la lecture que du texte lui-même ${ }^{14}$, on peut suggérer de regarder d'un peu près la fort étrange toponymie qui se met en place dans In the Country of Last Things. Car celle-ci, plutôt que d'offrir, comme souvent en science-fiction, un exotisme de bon aloi, obéit à un système beaucoup plus troublant qui fait que les noms de lieux, tout en étant familiers, ne désignent (du moins les premiers d'entre eux) aucun lieu connu d'aucun lecteur: Lampshade Road (p. 37, 46), Dictionary Place (p. 43, 53), Filament Square (p. 44, 53), Memory Avenue (p. 47, 56), et ainsi de suite. On voit que le problème ne touche pas aux connaissances géographiques du lecteur mais bien au curieux régime de nomination adopté dans la ville elle-même innommée -, régime qui consiste à recourir, pour une bonne part, au commun plutôt qu'au propre. Comment mieux signifier que ce qui distingue ce monde-là du nôtre n'est pas tant quelque événement catastrophique (jamais mentionné, de toute façon, lui non plus) qu'un bouleversement d'abord langagier? Comment mieux signaler que le propre, l'identitaire ont ici fait place au commun, à l'indifférencié ${ }^{15}$ ? De même, les

$13 C f$. respectivement p. 149,162 et $150,163$.

14 Mais qu'est-ce donc que le texte, demandait Ricardou voilà déjà une vingtaine d'années, considéré indépendamment de toute lecture?

15 Processus semblable dans le cas des partis politiques qui, pendant un temps, n'étaient désignés que par des couleurs (p. 135-136, 148). On peut bien entendu lire cela comme une allusion à l'indifférenciation grandissante des partis politiques, notamment américains. Mais on ne négligera pas pour autant l'autre allusion aux personnages de Gbosts: Blue, White, Black et Brown. 
différentes parties de la ville ne portent pas des noms mais des nombres - des nombres sans doute attribués par quelque administration puisqu'ils correspondent à autant de "zones de recensement", ce qui fait implicitement resurgir, non seulement l'idée de dénombrement mais aussi celle de nom ${ }^{16}$.

En ce qui concerne les toponymes eux-mêmes, on notera d'abord un net effet d'antiphrase, qui fait que cette ville sans plaisir ni espoir, où la mémoire collective s'effiloche (au point que même des mots comme "avion" semblent en passe de disparaître, voir p. 87, 97), exhibe des noms comme Circus Lane (p. 49, 59) ${ }^{17}$, Progress Avenue (p. 80, 91), Memory Avenue (p. 47, 56) et Dictionary Place (p. 43, 53). Mais on notera aussi de curieux courts-circuits qui permettent de recomposer, en un geste qui peut rappeler celui des "chasseurs d'objets", une lampe en porcelaine (China) avec son ampoule (Filament) et même son abat-jour (Lampshade).

Il est temps cependant de reconnaitre que les noms propres ne sont pas absents de la toponymie du roman. Au contraire, ils deviennent nettement majoritaires dans la seconde moitié du roman: Ptolemy Boulevard (p. 92, 103), Miro Avenue (p. 110, 121), Nero Prospect (p. 136, 148), Windsor Boulevard (p. 152, 165), Diogenes Terminal (p. 162,175$)^{18}$. Il n'est pas indifférent

16 On peut toutefois se demander, étant donné l'atrophie des responsabilités de l'administration, si cette appellation ne serait pas le vestige d'un recensement déjà ancien. Dans cette ville où on meurt beaucoup mais où aucun bébé ne naît, tout recensement, de toute façon, ne servirait guère qu'à rayer des noms de la liste précédente.

17 Ce toponyme offre en outre ceci d'intéressant qu'il peut aussi se lire comme un glissement de l'appellation générique (circus: rond-point) à l'appellation particulière: quelque chose comme une avenue Rue, donc, avec en prime une bizarre incompatibilité (comment se figurer une ruelle Rond-Point?). C'est aussi un jeu polysémique qui fera que China Street (p. 79, 89) ne renvoie pas forcément au pays mais peut-être à la porcelaine, l'insistance du commun sous le propre se faisant ici à la faveur d'une sorte de contamination onomastique. (La version de Patrick Ferragut escamote cette possibilité en procédant curieusement, pour une fois, à une traduction: "rue de Chine".)

18 Auxquels s'ajoutent deux toponymes communs: Turquoise Avenue (p. 151, 164) et Millenial Gate (p. 185, 200). Quant au début du roman, les noms propres n'en sont pas totalement absents puisqu'on y trouve un énigmatique Muldoon (p. 43, 53) qu'il est possible d'identifier comme étant Robert David Muldoon, premier ministre de la Nouvelle-Zélande entre 1975 et 1984 . Que son nom soit attribué à un boulevard pourrait constituer un indice en ce qui concerne la localisation géographique de la ville, si d'autres toponymes ne 
que cette liste, qui semble infirmer l'étiolement de la mémoire collective, apparaisse précisément à partir du moment où Anna a emménagé dans la Bibliothèque nationale ${ }^{19}$ (où d'ailleurs un groupe tente de rédiger un dictionnaire) et vit avec Samuel Farr, qui y écrit un livre qui devrait - aurait dû - constituer une immense somme sur la ville et son passé récent. Si la première moitié du roman est dominée par le motif de l'effacement, la seconde, en revanche, semble accréditer, au moins pendant un temps, la possibilité d'un sauvetage du passé (ainsi qu'un espoir pour l'avenir lorsqu'Anna devient enceinte). Cela dit, la liste donne l'échantillon d'une bien étrange mémoire: certes, Miró et Windsor sont des choix toponymiques plausibles ${ }^{20}$, mais Ptolémée l'est passablement moins et Néron pas du tout - à moins que des bouleversements culturels dont nous ne pouvons que soupçonner l'ampleur aient amené quelque édile à commémorer le souvenir d'un astronome aux théories discréditées depuis des siècles et celui d'un empereur romain surtout célèbre pour ses crimes - à commencer par celui d'avoir peut-être partiellement détruit une ville.

Mais, s'il est irrécusable qu'on peut assigner à la liste des motivations fictives qui en font une série d'indices aidant à reconstruire (fragmentairement) le monde fictif, on ne saurait pour autant la réduire à ce rôle somme toute documentaire. Ce serait singulièrement se méprendre sur l'onomastique austé-

venaient pas problématiser - j'y reviens tout de suite - une telle lecture indicielle, sans l'interdire totalement cependant. Quoi qu'il en soit, la Nouvelle-Zélande, antipode par excellence, constitue un emblème tout à fait convenable, aussi bien de l'éloignement que du renversement.

19 On aura remarqué la minuscule qui, dans la traduction française, remplace la majuscule de l'original: "National Library". Il n'est pas difficile de supposer que cette minuscule vise surtout à distinguer cette bibliothèque de la réelle Bibliothèque Nationale. Cet exemple montre clairement à quel point les cadres de référence peuvent changer d'une lecture à l'autre: pour un lecteur nord-américain familier de la prédilection des récits d'Auster pour la ville de New York, c'est plutôt la substitution Public/National qui peut venir à l'esprit, et donc un obstacle à une pourtant tentante identification de la ville avec New York.

20 Sur un autre plan, "Windsor" s'insère dans l'insistant réseau onomastique constitué autour de la royauté: Anna vit quelques mois avec un couple formé d'une Isabel et d'un Ferdinand, Woburn House est dirigée par une Victoria (Woburn) et emploie les services d'un Boris (Stepanovich), sans compter, on l'a vu, qu'il est brièvement question d'un Quinn qui à la faveur d'une légère déformation phonétique peut s'entendre: Queen. 
rienne, en effet, que de la ramener à un rôle simplement indiciel - non parce que les récits d'Auster feraient fi de la référence, mais bien parce que cette référence n'est jamais si simple qu'elle permette d'ignorer les réseaux du texte, des textes.

Ainsi, une fois reconstituée, la série des noms propres, Ptolémée-Miró-Néron-Windsor-Diogène, apparaît scander en pointillés les étapes de l'histoire d'Anna: les nuits passées à observer les étoiles en fumant avec Sam (p. 109, 121), l'incendie qui détruit la bibliothèque ( $p .129-130,141-142$ ), la vie de château à Woburn House, la vie d'ermite de Sam au Terminal Diogène (p. 162, 175) ${ }^{21}$. Du coup, les noms propres ne se réduisent plus à des indices d'une mémoire collective (de toute façon énigmatique), mais deviennent autant de fragments de synopsis, de récits réinjectés dans celui que nous lisons.

\section{L'enchâssement fantôme}

Si l'examen de la toponymie montre à quel point Auster remet en question, mine de rien, certains poncifs de la sciencefiction - à commencer par son exotisme finalement rassu$\operatorname{rant}^{22}$ - un coup d'œil sur les stratégies énonciatives montrera que cette remise en question est plus radicale encore. Pour saisir l'étendue de ce bouleversement, il faut d'abord rappeler que la science-fiction a fait - et fait encore - usage de modèles discursifs repris du récit de voyage. Cet usage sert des fins essentiellement vraisemblabilisantes; comme la science-fiction met en place des mondes fictifs divergeant du monde de référence des lecteurs, le modèle du récit de voyage permet de motiver l'inser-

21 On pourra cependant remarquer, d'une part, que la fiction et la série onomastique ne coïncident qu'en ce dernier point (dans les autres cas, les toponymes apparaissent au milieu d'autres épisodes) et que, d'autre part, rien dans ma liste ne correspond à Miró. Ce décalage et cette lacune n'ont pas tant pour effet, je crois, d'invalider l'hypothèse que je propose, que de montrer qu'elle est précisément une hypothèse, et donc un résultat conjoint de l'écriture et de la lecture (et même de la relecture). Ainsi, il est tout à fait possible que, pour un lecteur ou une lectrice plus familier avec l'œuvre de Miró, tel épisode puisse être mis en contact avec, par exemple, tel titre de toile. (Pour plus de détails sur ces questions, on se reportera à mon article "La lecture erratique" in Denis Saint-Jacques (dir.), Comment lit-on?, Québec, Nuit Blanche éditeur, coll. "Les cahiers du CRELIQ •, 1994.)

22 Ce qu'avait déjà remarqué Michel Butor dans "La crise de croissance de la science-fiction", repris in Répertoire, Paris, Minuit, 1960, p. 189. 
tion, au sein du discours narratif, de larges segments didactiques. On ne s'étonnera donc pas de sa fortune ${ }^{23}$.

À première vue, In the Country ne semble pas faire exception à cet égard: le texte se présente comme une longue lettre dans laquelle Anna décrit minutieusement à son destinataire la ville étrangère où elle est venue à la recherche de son frère disparu. Mais cette convergence se révèle, à bien y regarder, superficielle: alors que les récits de voyage science-fictionnels s'emploient à neutraliser l'écart entre le savoir encyclopédique du narrataire et celui des lecteurs réels (de façon à ce qu'aucun savoir partagé avec le premier mais non avec les seconds ne vienne compromettre ou même seulement compliquer la compréhension), la lettre d'Anna, au contraire, présuppose quantité de données - à commencer par sa situation géographique, l'époque où elle vit, et la nature des événements qui ont mené à l'état actuel des choses - au point où il est impossible de déterminer, par exemple, si l'effondrement de la société civile se limite à la ville - ou peut-être au pays qu'elle habite, ou si des chambardements d'une tout autre ampleur n'ont pas eu lieu. Cette "stratégie de la présupposition" s'étend jusqu'à l'identité de la narratrice, qui demeure longtemps imprécisée: ce n'est qu'après plusieurs dizaines de pages (p. 49,59) qu'on apprendra qu'elle s'appelle Anna, et plus loin encore (p. 101, 110) que son nom de famille est Blume ${ }^{24}$.

Je viens de qualifier Anna de "narratrice", mais nous savons que ce n'est pas à proprement parler le cas, même si, paradoxalement, la quasi totalité des phrases qui composent le roman sont bien énoncées par elle. C'est que la lettre d'Anna est enchâssée par une instance narrative parfaitement anonyme et dont nous ne savons strictement rien, pour la simple raison que cette

23 D'autant plus qu'il n'est pas rare que la science-fiction adopte un optimisme épistémique (qui n'est guère plus de mise en sciences), et qui fait que des personnages fraîchement débarqués sur des planètes ou dans des sociétés inconnues semblent n'éprouver aucune difficulté à s'en rendre intelligible les particularités, le fonctionnement et même la langue. À cet égard le pessimisme de Stanislas Lem est tout à fait bienvenu, non seulement sur le plan épistémique mais aussi en ce qui concerne les stratégies discursives ainsi rendues possibles. (Voir par exemple ses romans L'invincible, Eden, Solaris, Fiasco et La voix du maître.)

24 Ce dispositif est bien entendu saboté dans la version française en raison du titre qui livre d'emblée le nom complet d'un personnage qu'on n'a aucune peine à identifier avec la narratrice. 
instance narrative n'apparaît qu'à la faveur d'une poignée d'incises: d'abord quatre en quelques pages, puis une autre une trentaine de pages plus loin ${ }^{25}$, puis plus rien, selon une disparition progressive qu'on pourra comparer à celle du Chat de Cheshire ou, plus près de In the Country parce que reposant aussi sur un dispositif énonciatif, celle du fameux "nous" de Madame Bovary ${ }^{26}$.

Mais les incises de In the country se distinguent du "nous" de Madame Bovary en ce que, si le second renvoie à une instance fictive déterminée, bien que problématique - les camarades de classe de Charles -, les incises, elles, demeurent, comme je l'ai indiqué, parfaitement anonymes. L'enchâssement de la lettre d'Anna, tout en étant narratologiquement irrécusable, n'est, discursivement parlant, qu'un enchâssement fantôme: l'instance narrative garde le silence non seulement sur elle-même et sur sa situation (notamment temporelle et géographique, on y reviendra), mais aussi sur ses relations à Anna Blume et à sa missive. $\mathrm{Au}$ point que même l'hypothèse qui y verrait le destinataire (jamais nommé) de la lettre d'Anna n'est qu'une pure conjecture, plausible mais nullement assurée.

Par ailleurs, le fait que l'instance narrative n'apparaisse qu'à la faveur d'incises occasionne une situation un peu paradoxale, dans la mesure où ce qui narrativement enchâsse se trouve, sur le plan syntaxique, enchâssé. Cette contradiction aurait pu rester anodine, mais In the Country l'exacerbe en évidant la narration extradiégétique, qui se trouve ainsi totalement absentée, à l'exception justement de ces minuscules agrafes discursives que sont les incises. Du coup, l'hypertrophie du discours enchâssé ne souligne que mieux la quasi disparition élocutoire du discours enchâssant - et, avec, lui, de son improbable instance énonciative. Le seul dire en effet qui appartient en propre au narrateur (le masculin, ici, est déjà une supposition) est celui par lequel il délègue l'énonciation à Anna Blume. Bref: il ne prend la parole

25 "Ce sont les dernières choses, a-t-elle écrit" (p. 9); "C'est ainsi que je vis, poursuivait-elle dans sa lettre" (id.); "Il y a des gens qui sont si minces, écrivait-elle, qu'il leur arrive d'être emportés par le vent " (p. 11); “Lorsque tu marches dans la rue, poursuivait-elle, tu dois te souvenir de ne faire qu'un pas à la fois" (p. 13); "Je n'ai jamais trouvé William, a-t-elle continué" (p. 48). C'est chaque fois moi qui souligne.

26 J'analyse ce dispositif dans Châteaux de pages: la fiction au risque de sa lecture, Montréal, Hurtubise $\mathrm{HMH}$, coll. "Brèches", 1994. 
que le temps de dire qu'elle dit - ou plutôt écrit. De lui, nous ne saurons rien: ni son identité (est-il le destinataire de la missive d'Anna? quelqu'un qui l'aurait trouvée - à la suite de quels hasards, de quelles péripéties?), ni sa situation géographique (habite-t-il la même ville qu'Anna? une autre partie du pays? ou alors le pays qu'elle a quitté, de l'autre côté de l'océan?) ou temporelle (est-il un contemporain d'Anna? les amateurs de science-fiction pourront aisément imaginer, au contraire, qu'une période considérable les sépare peut-être, de telle sorte que l'instance narrative appartiendrait à un futur encore plus lointain et encore plus inconcevable). Rien ne prouve d'ailleurs que le narrateur soit même un habitant du monde fictif d'Anna; qu'est-ce qui dit qu'il n'a pas plutôt imaginé ce qu'il feint de rapporter? Nous pourrions multiplier les questions, mais celles-ci ne feraient que poser obliquement celle-ci: qu'est-il, sinon une hypothèse, une rêverie peut-être, de la lecture?

Ces questions ne touchent pas que l'aspect énonciatif du texte: comme maints textes d'Auster, In the Country exploite, à sa façon à la fois simple et subtile, les effets fictionnels de l'énonciation. Nulle part ceci n'est aussi spectaculaire - et troublant que dans les dernières lignes du texte:

La seule chose que je demande à présent, c'est la chance de vivre un jour de plus. C'est Anna Blume, ta vieille amie d'un autre monde. Lorsque nous arriverons là où nous allons, j'essaierai de t'écrire à nouveau, je te le promets. (p. 202)

Le texte parvient ainsi à capturer la blancheur qui l'enrobe, à l'intégrer dans sa fiction - mais une fiction qui se trouve ainsi éclater en un faisceau de conjectures: Anna et ses amis ont-ils été arrêtés en essayant de sortir de la ville? ont-ils survécu? Anna a-t-elle été empêchée d'écrire pour d'autres raisons? a-t-elle écrit une autre lettre, qui ne serait jamais parvenue à son destinataire? à l'instance narrative?

La fiction apparaît ainsi, au moment où elle est sur le point de se loger dans la mémoire du lecteur, comme un fragile édifice qui surgit de la blancheur de la page. Aussi est-on tenté de conclure en citant cette description de Boris Stepanovich, l'homme aux chapeaux pseudonymiques et aux identités fluctuantes: "Il utilisait le langage comme un moyen de locomotion" (p. 158). In the Country montre qu'il est aussi, ce faisant, un moyen de disparition. 